\section{Starch safety in resuscitation}

To the Editor: The Western Cape Department of Health (WC $\mathrm{DoH}$ ) has taken a decision to withdraw all intravenous fluids (IVFs) containing hydroxyethyl starch (HES) from hospitals in the Western Cape ${ }^{[1]}$ with similar action contemplated in the Free State and Gauteng. This was in response to recommendations from:

- The European Medicines Agency's Pharmacovigilance Risk Assessment Committee (EMA PRAC) that HES IVFs be withdrawn from clinical use. ${ }^{[2]}$

- The United Kingdom Medicines and Healthcare Products Regulatory Agency (MHRA) which has issued a recall of all HES IVFs in the UK. ${ }^{[3]}$

- The United States Food and Drug Administration ${ }^{[4]}$ which advises that HES IVFs be used with caution in ICU, cardiac surgery and patients with known kidney disease or coagulopathy. Further advice was that HES should be stopped if coagulopathy or renal dysfunction develops, as well as that renal function should be monitored for 90 days after HES administration.

The actions of the WC DoH could be justified if the recommendations from the regulators in Europe, the USA and UK were based on relevant scientific evidence. Unfortunately, the evidence provided is not only flawed, but also has been applied to clinical scenarios not included in the studies used in evidence. Regarding these studies, HES IVFs were administered to critically ill patients with sepsis. The oldest study ${ }^{[5]}$ from 2008, used a hyperoncotic (10\%) solution of HES 200/0.5 that is no longer used in South Africa and differs significantly from the HES IVFs currently in use. This caveat applies equally to the recent metaanalysis of starches in JAMA where the majority of starches used were outdated or hyperoncotic or used in unnecessary volumes over prolonged periods of time. ${ }^{[6]}$ Subsequent studies include $6 \mathrm{~S}^{[7]}$ from Scandinavia and CHEST $^{[8]}$ from Australasia, which were published in 2012.

The $6 \mathrm{~S}^{[7]}$ trial used a potato-derived 6\% (iso-oncotic) HES 130/0.4 (Venofundin, B Braun) IVF and compared this with Ringer's acetate solution. The study involved 798 patients with an average age of 65 . Renal replacement therapy (RRT) was used more frequently in the HES group ( $22 \%$ v. $16 \%$; $p=0.04)$. However, there was no significant difference in the number of patients with a doubling of creatinine levels, and only one patient in each group was dialysis-dependent at day 90 . This finding could be explained by the lack of a protocol for RRT. There was a significant difference in 90 -day mortality $(51 \% \mathrm{v}$. $43 \%$; $p=0.03$ ). This study may therefore be summarised as a trial of a potato-derived HES IVF v. Ringer's acetate in an elderly population of critically ill septic patients that, although showing a significant increase in use of RRT and 90-day mortality, might have been influenced by lack of RRT protocols.

The $\mathrm{CHEST}^{[8]}$ trial used a waxy maize-derived 6\% (iso-oncotic) HES 130/0.4 (Voluven, Fresenius-Kabi) IVF compared with $0.9 \%$ saline. The study involved 6651 patients with an average age of 63 . Patients who were included had been admitted to the ICU for more than 10 hours and received an average of 3.51 of other fluids before first receiving study fluid. Lactate and base deficit data suggest that these patients were already fluid resuscitated on entry to the study. The administration of any further volume expander would not seem to have been appropriate in these cases. RRT was used more frequently in the HES group ( $7.0 \%$ v. $5.8 \%$; $p=0.04$ ) but renal injury occurred more commonly in the saline group $(38.0 \%$ v. $34.6 \%$; $p=0.005$ ). The incidence of renal failure was similar in the two groups, at $10.4 \%$ and $9.2 \%$ respectively ( $p=0.12$ ), which did not correlate with the increased use of RRT in the HES group. An additional problem was that, prior to randomisation, HES IVF was given to 509 patients in the HES group and 508 patients in the saline group.

Importantly, there was a significant increase in new cardiovascular failure in patients receiving saline $(39.9 \%$ v. $36.5 \%$; $p=0.03)$ but more blood products were used in the HES group.

The CHEST trial may be summarised as a trial of HES IVF compared with $0.9 \%$ saline in an elderly population of critically ill, septic patients that showed a significant increase in use of renal replacement therapy (but with no difference in mortality at 28 or 90 days), less renal injury and similar rates of renal failure.

Taken together, the two most recent studies ${ }^{[7,8]}$ indicate, at most, that HES is associated with an increased risk of renal replacement therapy in elderly, critically ill septic patients. The risk of mortality is less clear, with only the $6 \mathrm{~S}$ study showing an increase in 90 - but not 28-day mortality, and the substantially larger CHEST study showing no difference.

None of the studies used by the regulatory agencies addressed the use of HES IVF in patients with trauma or those undergoing major elective or emergency surgery for non-septic disease. One of the few randomised control trials of HES IVF compared with $0.9 \%$ saline was undertaken in Cape Town. ${ }^{[9]}$ A total of 109 patients was studied, with the 67 patients who suffered penetrating trauma requiring more saline (7.4 1 v. 5.11 ; $p<0.001$ ). Renal injury occurred more frequently in the saline group than the HES group ( $16 \%$ v. $0 \%$; $p=0.018$ ).

Major trauma or surgery where transfusion is likely is most appropriately managed by early administration of blood and blood products to maintain oxygen delivery and limit coagulopathy. The role of clear fluids, either crystalloid or colloid, is limited where transfusion is required. ${ }^{[10]}$ However, a substantial number of patients suffering trauma or undergoing elective or emergency surgery require intravascular volume replacement but not transfusion.

Caesarean section is an example of a procedure requiring volume loading owing to spinal anaesthesia and blood loss, where transfusion is seldom necessary. A recent meta-analysis has demonstrated the efficacy of colloid solutions, including HES IVF for this indication. ${ }^{[1]]}$

Enhanced recovery programmes for major elective surgery, such as colectomy, also advocate the use of colloids such as HES IVF for replacement of intraoperative blood loss in preference to crystalloids, which have a greater potential to cause bowel oedema, leading to ileus and anastomotic dehiscence. ${ }^{[12]}$

Alternatives in such situations (including crystalloids, gelatin solutions, blood products such as albumin or plasma) are limited. Crystalloids are associated with development of peripheral and organ oedema increasing the incidence of abdominal compartment syndrome, cardiac and renal failure and exacerbating the acute respiratory distress syndrome. ${ }^{[13]}$

Gelatin solutions are associated with a risk of anaphylaxis and have minimal advantages over crystalloids in terms of intravascular persistence. ${ }^{[14]}$ Blood products are expensive, are not available in large volumes and should only be administered for specific indications. ${ }^{[15]}$

The authors appeal for a more rational thought process about the use of HES IVFs. Owing to possible renal harm in elderly, critically ill patients with sepsis, HES IVFs should be withheld from them. There is, however, no evidence that HES IVFs should be withheld from non-septic patients without critical illness who require intravascular volume replacement, but not transfusion. HES IVFs for this indication should be retained for use by anaesthesiologists, emergency physicians and intensivists.

Conflict of interest. Drs Hodgson, Spruyt and Gopalan and Proff. Richards and Dickerson received speaker honoraria from Fresenius Kabi. 


\section{CORRESPONDENCE}

\section{R E Hodgson}

Inkosi Albert Luthuli Central Hospital, Durban, and Department of Anaesthesia, Nelson R Mandela School of Medicine, University of KwaZulu-Natal, Durban, South Africa richard.hodgson@ialch.co.za

\section{G A Richards}

Division of Pulmonology and Critical Care, Charlotte Maxeke Johannesburg Academic Hospital, and University of the Witwatersrand, Johannesburg, South Africa

\section{A C Lundgren}

Department of Anaesthesiology, University of the Witwatersrand, and Department of Anaesthesia, Chris Hani Baragwanath Academic Hospital, Johannesburg, South Africa

\section{G L Spruyt}

Division of Critical Care, Bloemfontein Academic Complex, Universitas Hospital, Bloemfontein, South Africa

\section{J P Pretorius}

Department of Critical Care, Medical School, Faculty of Health Sciences, University of Pretoria, and Steve Biko Academic Hospital, Pretoria, South Africa

\section{R Mathiva}

Division of Critical Care Medicine, Chris Hani Baragwanath Academic Hospital and University of the Witwatersrand, Johannesburg, South Africa

\section{R Dickerson}

Department of Emergency Medicine, Chris Hani Baragwanath Academic Hospital and University of the Witwatersrand, Johannesburg, South Africa

\section{P D Gopalan}

Department of Anaesthesia, Nelson R Mandela School of Medicine, University of KwaZulu-Natal, Durban, South Africa

1. Hayes H. Suspension of use of infusion solutions containing hydroxyethyl-starch at Western Cape Government healthcare facilities until further notice. Circular H114/2013. 5 July 2013.

2. European Medicines Agency's Pharmacovigilance Risk Assessment Committee. PRAC recommends suspending marketing authorisations for infusion solutions containing hydroxyethyl-starch. EMA/349341/2013. 14 June 2013.

3. Medicines and Healthcare Products Regulatory Agency. Hydroxyethyl starch intravenous infusion: Suspension of licences. http://www.mhra.gov.uk/ Safetyinformation/DrugSafetyUpdate (accessed 14 July 2013).

4. FDA Safety Communication: Boxed warning on increased mortality and severe renal injury, and additional warning on risk of bleeding, for use of hydroxyethy starch solutions in some settings. http://www.fda.gov/BiologicsBloodVaccines/ SafetyAvailability (accessed 14 July 2013).

5. Brunkhorst FM, Engel CE, Bloos F, et al. for the German Competence Network Sepsis (SepNet). Intensive insulin therapy and pentastarch resuscitation in severe sepsis. N Engl J Med 2008;358:125-139. [http://dx.doi.org/10.1056/NEJMoa070716]

6. Zarychanski R, Abou-Setta AM, Turgeon AF, et al. Association of hydroxyethyl starch Zarychanski R, Abou-Setta AM, Turgeon AF, et al. Association of hydroxyethyl starch
administration with mortality and acute kidney injury in critically ill patients requiring administration with mortality and acute kidney injury in critically ill patients requiring
volume resuscitation: A systematic review and meta-analysis. JAMA 2013;309(7):678volume resuscitation: A systematic review and
688 . [http://dx.doi.org/10.1001/jama.2013.430]

7. Perner A, Haase N, Guttormsen AB, et al. for the $6 \mathrm{~S}$ Trial Group and the Scandinavian Critical Care Trials Group. Hydroxyethyl starch 130/0.4 versus Ringer's acetate in severe sepsis. N Engl J Med 2012;367(2):124-134. [http://dx.doi.org/10.1056/NEJMoa 1204242]

8. Myburgh JA, Finfer S, Bellomo R for the CHEST Investigators and the Australian and New Zealand Intensive Care Society Clinical Trials Group. Hydroxyethyl starch or saline for fluid resuscitation in intensive care. N Engl J Med 2012;367(20):1901-1911. [http://dx.doi.org/10.1056/NEJMoa1209759]

9. James MF, Michell WL, Joubert IA, et al. Resuscitation with hydroxyethyl starch improves renal function and lactate clearance in penetrating trauma in a randomized controlled study: The FIRST trial (Fluids in Resuscitation of Severe Trauma). Br J Anaesth 2011;107(5):693-702. [http://dx.doi.org/10.1093/bja/aer229]

10. Duchese JC MCSwain NE Jr Cotton BA, et al. Dame control resuscitaion I Trauma Injury Infect Crit Care 2010;69(4):976-990. [http://dx.doi.org/10.1097 Trauma Injury Infect
TA.0b013e3181f2abc9]

11. Li L, Zhang Y, Tan Y, Xu S. Colloid or crystalloid solution on maternal and neonatal hemodynamics for cesarean section: A meta-analysis of randomized controlled trials. Obstet Gynaecol Res 2013;39(5):932-941. [http://dx.doi.org/10.1111/jog.12001]

12. Abraham-Nordling M, Hjern F, Pollack J, Prytz M, Borg T, Kressner U. Randomized clinical trial of fluid restriction in colorectal surgery. Br J Surg 2012;99(2):186-191 [http://dx.doi.org/10.1002/bjs.7702]

13. Bihar Si, Prakash S, Bersten AD. Post resuscitation fluid boluses in severe sepsis or septic shock: Prevalence and efficacy (PRICE study). Shock 2013;40(1):28-34. [http://dx.dol. org/10.1097/SHK.0b013e31829727fl]

14. Saw MM, Chandler B, Ho KM. Benefits and risks of using gelatin solution as a plasm expander for perioperative and critically ill patients: A meta-analysis. Anaesth Intens Care Med 2012;40(1):17-32.

15. Groeneveld AB, Navickis RJ, Wilkes MM. Update on the comparative safety of colloids A systematic review of clinical studies. Ann Surg 2011;253(3):470-483. [http://dx.doi org/10.1097/SLA.0b013e318202ffoo] 
To the Editor: The article ${ }^{[1]}$ by Parrish and Blockman in the June 2013 SAMJ requires a carefully considered, unbiased and scientific response, as much of the evidence that relies almost exclusively on 3 flawed studies is not as strong as these authors would have us believe.

It is profoundly disappointing to see that the authors rely heavily on the VISEP study ${ }^{[2]}$ and meta-analyses that include it as evidence of starch-associated renal damage. The VISEP study employed prolonged infusions of hyperoncotic starch with inadequate crystalloid support. It has been known for over 30 years that repetitive dosing with hyperoncotic colloids will damage the kidney, irrespective of colloid type. Given these conceptual and ethical flaws, VISEP, and any meta-analyses that include it, should not be quoted as evidence of direct nephrotoxicity or mortality associated with isooncotic tetrastarches.

The CHEST trial ${ }^{[3]}$ to which the authors refer was powered for a single endpoint: mortality. The result shows that the starch tested in this study does not increase mortality compared with saline. The secondary conclusion (that there was an increase in the rate of renal replacement therapy) lacks internal validity. After adjustment for known covariates, this random observation ceased to be statistically significant. No criteria were specified for the initiation of renal replacement therapy. RIFLE criteria $\mathrm{R}$ and I contradict the finding and, in fact, favour HES. The incidence of renal failure, and the number of dialysis days (both more robust indicators of renal injury) were not different between the groups. The only valid conclusion from CHEST is that the HES product used is not associated with increased mortality.

In the $6 \mathrm{~S}$ study, ${ }^{[4]}$ markers of tissue perfusion (lactate, central venous oxygen saturation and central venous pressure) were normal at study entry, indicating that resuscitation had been completed before enrolment. However, in excess of 4.51 fluid was administered on day 1 , a trend sustained for the next 3 days. Such fluid excess has been associated with intensive care mortality and may account for the high overall renal injury and mortality reported. These and other criticisms have been raised by others in correspondence with the NEJM. It is also important to note that the potato-based starch product used in $6 \mathrm{~S}$ is a very different chemical entity from the corn starch-based version.

Exclusion of the initial resuscitation phase in these studies is a fatal flaw; repetitive fluid boluses, after initial resuscitation, have been shown to be useless and potentially harmful. ${ }^{[5]}$ The only indication for the administration of colloids is hypovolaemia, but none of these studies demonstrated that the patients were hypovolaemic. The administration of colloids in the presence of normal or increased plasma volume damages the endothelial glycocalyx, resulting in increased transudation into the interstitium. ${ }^{[6]}$ Fluid overload is undeniably easier to achieve with inappropriately administered colloid than with similar crystalloid volumes. The one incontrovertible fact in the fluid debate is that excessive fluid administration is harmful.

A serious weakness of the Parrish and Blockman paper is the extrapolation of these intensive care data to other fields of resuscitation, particularly those where volume depletion is likely. The largest proportion of colloid administration $(>80 \%)$ is in trauma and perioperative settings, yet almost all publications in this area have been ignored. Systematic reviews have found no evidence whatsoever of renal injury associated with the use of modern HES. ${ }^{[7]}$ Recent trauma studies have suggested that the use of colloids in acute resuscitation is probably beneficial. ${ }^{[8]}$

The most significant recent advances in perioperative fluid therapy have included crystalloid restriction ${ }^{[9]}$ with reliance on colloid volume support against robust markers of volume deficiency. Meta-analysis of the use of dynamic monitoring to guide colloid administration clearly showed benefit in terms of reduction in complications and hospital stay. ${ }^{[10]}$
The authors' article, and recent moves to withdraw HES, have ignored the serious criticisms of studies that favour their hypothesis and have not considered the peri-operative data that uniformly favour colloid use.

In summary, what have we really learned from the recent data? The unrestricted use of colloids to treat such nonvolumetric markers as central venous pressure, hypotension and inadequate urine output, particularly in the later stages of critical illness, is inappropriate and potentially harmful, and should be abandoned. Where clear markers of hypovolaemia are present, colloids appear to be superior to crystalloids for initial resuscitation. There is no evidence of harm from any of the modern colloids, including HES (apart from anaphylaxis, particularly with the gelatins), when these solutions are used for the appropriate indications.

Alternative strategies to the use of synthetic colloids are all problematic. Albumin is simply too expensive. Increased use of crystalloid for resuscitation has consistently been shown to be harmful, ${ }^{[1]]}$ with far stronger indicators of harm than those produced by Parrish and Blockman. Increased use of blood and blood products is equally unsound. Whilst the use of fluids in patients in ICU, and in those with sepsis in particular, clearly needs to be re-assessed, the perioperative evidence suggests that abandoning HES would do substantially more harm than good. Those who advocate this must bear the burden of proof that current practice is flawed. There is no such evidence in the Parrish and Blockman article.

Space constraints prevent a fuller development of these arguments, but all opinions expressed are supported by a full range of references that are available on request.

M F M James

I A Joubert

J L Piercy

Department of Anaesthesia, University of Cape Town, South Africa

mike.james@uct.ac.za

\section{A Levin}

Department of Anaesthesia and Critical Care, University of Stellenbosch, South Africa

1. Parrish A, Blockman M, Starch safety in resuscitation - when will we ever learn? S Afr Med I 2013;103(6):365-367. [http://dx.doi.org/10.7196/SAMJ.6969]

Brunkhorst FM, Engel C, Bloos F, et al. Intensive insulin therapy and pentastarch resuscitation in severe sepsis. N Engl J Med 2008;358(2):125-139. [http://dx.doi.org/10.1056/NEJMoa070716]
s.

3. Myburgh JA, Finfer S, Bellomo R, et al. Hydroxyethyl starch or saline for fluid resuscitation in intensive Myburgh JA, Finfer S, Bellomo R, et al. Hydroxyethyl starch or saline for fluid resuscitation in
care. N Engl J Med 2012;367(20):1901-1911. [http://dx.doi.org/10.1056/NEJMoa 1209759]

care. N Engl J Med 2012;367(20):1901-1911. [http://dx.doi.org/10.1056/NEJMoa1209759]
4. Perner A, Haase N, Guttormsen AB, et al. Hydroxyethyl starch 130/0.42 versus Ringer's acetate in severe sepsis. N Engl J Med 2012;367(2):124-134. [http://dx.doi.org/10.1056/NEJMoa1204242

5. Bihari S, Prakash S, Bersten AD. Post resusicitation fluid boluses in severe sepsis or septic shock: prevalence and efficacy (price study). Shock 2013;40(1):28-34. [http://dx.doi.org/10.1097/ SHK.0b013e31829727f1]

6. Chappell D, Jacob M, Hofmann-Kiefer K, Conzen P, Rehm M. A rational approach to perioperative fluid management. Anesthesiology 2008;109(4):723-740. [http://dx.doi.org/10.1097/ ALN.0b013e3181863117]

Van der Linden P, James MF, Mythen M, Weiskopf RB. Safety of modern starches used during surgery. Anesth Analg 2013;116(1):35-48. [http://dx.doi.org/10.1213/ANE.0b013e31827175da]

8. James MF. Place of the colloids in fluid resuscitation of the traumatized patient. Curr Opin Anaesthesiol 2012;25(2):248-252. [http://dx.doi.org/10.1097/ACO.0b013e32834fcede]

9. Brandstrup B, Svendsen PE, Rasmussen M, et al. Which goal for fluid therapy during colorectal surgery 9. Brandstrup B, Svendsen PE, Rasmussen M, et al. Which goal for fluid therapy during colorectal surgery
is followed by the best outcome: near-maximal stroke volume or zero fluid balance? Br J Anaesth is followed by the best outcome: near-maximal stroke volu

10. Hamilton MA, Cecconi M, Rhodes A. A systematic review and meta-analysis on the use of preemptive hemodynamic intervention to improve postoperative outcomes in moderate and high-risk surgical patients. Anesth Analg 2011;112(6):1392-1402. [http://dx.doi.org/10.1213/ANE.0b013e3181eeaae5]

11. Balogh Z, McKinley BA, Cocanour CS, et al. Supranormal trauma resuscitation causes more cases of abdominal compartment syndrome. Arch Surg 2003;138(6):637-642.

Parrish and Blockman respond: We are delighted that our article has created the opportunity for constructive debate on this topic. Our key point was that in the areas where published evidence about HES is most robust, there are signals of harm; and these signals are best identified by combining information from numerous high-quality sources. We also noted that there was insufficient evidence on HES 
use in other contexts to draw definitive conclusions about safety, and that it might be prudent to restrict usage until these concerns have been resolved.

Hodgson et al. note the recent international regulatory authority activity about HES products. The European Medicines Agency's Pharmacovigilance Risk Assessment Committee concluded that the risks of HES products outweigh their benefits, and recommended suspending their marketing authorisation. The UK's MHRA has withdrawn the products from the market via a Class 2 Medicines Recall, after the UK Commission on Human Medicines concluded that the products' benefits no longer outweigh their risks. The FDA has stated that HES solutions should not be used in critically ill adult patients, including patients with sepsis and those admitted to the ICU, and concluded that a Boxed Warning is warranted. A subsequent position statement ${ }^{[1]}$ by UK intensivists, anaesthetists and emergency medicine physicians supports the use of crystalloids as primary resuscitation fluids, in line with the provisional NICE guidelines on intravenous fluid therapy. ${ }^{[2]}$

International regulators do not make such decisions lightly, and contrary views need to be backed by meticulous reasoning. We are underwhelmed by the arguments put forward in the correspondence, and are concerned by what at times seems highly selective and in some cases simply erroneous interpretations of relatively straightforward trial evidence.

The letters from James et al. and Hodgson et al. share similar points: that three major trials (VISEP, CHEST and 6S), published in internationally respected journals, are flawed or lack external validity, and that there is sufficient evidence on HES use in other situations to pronounce it safer than crystalloid for these indications. A third topic concerns choice of alternative fluids and was not the focus of our article, but is worth comment.

We note that most of the objections to the three trials have been raised previously by industry-affiliated commentators, and competently answered in each journal's correspondence section by the particular trial's authors. We see little need to revisit all these issues here, but will make the general point that the systematic analysis of a body of literature accepts that very few trials are devoid of retrospectively identifiable flaws, but important trends may still emerge. We are fully aware of the issues with VISEP, and mentioned them in the article. We did not 'rely heavily' on this trial, and in fact it was not part of two of the three recent meta-analyses demonstrating renal harm. In the third meta-analysis (Zarychanski), it contributed $7.6 \%$ of the weight of the mortality analysis and $18 \%$ for renal harm.

Because mortality in CHEST was lower than expected (17.5\% rather than $26 \%$ ), even this very large trial was effectively underpowered to provide robust efficacy evidence on its own, which is where the utility of metaanalysis becomes apparent. Regarding the assertion that CHEST was not powered to assess acute kidney injury, we draw attention to the results of the authors' power calculation for acute kidney injury which are provided in the statistical methods section of the original paper $(90 \%$ power to detect a $1.5 \%$ difference from baseline of $6 \%$, with alpha 0.05.) The observation that 'once adjusted for 
known covariants this random observation (need for renal replacement therapy) ceased to be statistically significant' is open to alternative explanation: the relative risk (RR) for renal replacement therapy before adjustment was 1.21 (95\% confidence interval (CI) 1.00 - 1.45, including 1 because of the rounding process); after adjustment, it was essentially unchanged (RR $1.20,95 \%$ CI $1.00-1.44$ ). This is what is expected in a large, adequately randomised trial. Overall, the failure to demonstrate a mortality advantage (efficacy) in CHEST is precisely our point - there is concern about renal harm with no clear countervailing benefit on key patient-relevant clinical endpoints.

An error in interpretation of $6 \mathrm{~S}$ originally made by Wise et al., ${ }^{[3]}$ and corrected in the same NEJM correspondence section by the trial authors, has been repeated. Hodgson et al. state: 'but randomisation erred in that more patients receiving HES had septic shock and AKI at randomisation (179/336 v. $148 / 337)$ and (72/142 vs. $63 / 140)$ respectively. The figures come from a pre-defined subgroup analysis which showed that, of those patients presenting with shock at randomisation, 179 died in the HES group, and 148 in the crystalloid group. The two groups were evenly matched, with $84 \%$ of both groups presenting with shock (336 v. 337 patients.)

The second issue concerns peri-operative/surgical use of HES. James et al. refer to two reviews in support of their contention of proven safety; both were funded by the product manufacturers, and were the reason for our general caveats about interpreting such literature. We remind readers that, to be reasonably cautious about interpreting 'lack of harm' information, very large patient numbers are required for rare outcomes, follow-up needs to be for months and not days, and endpoints should be patient-relevant and not surrogate physiological markers. While both reviews allude to thousands of patients, $t$ the majority in fac are from trials where the comparator was another colloid.

Demonstrating that a newer colloid is less dangerous than an older one with recognised safety issues is unhelpful when the core issue is safety relative to carefully titrated crystalloids. We commented on this issue pertaining to the Martin review, and are puzzled by James et al's insistence on quoting it without addressing our reservations. In the published high-quality meta-analyses of HES use in critical illness, the numerator (number of events) required to generate a signal of renal harm was of the order of $300-400$ in each arm. In the surgical literature, numerators are still an order of magnitude lower (appropriate crystalloid comparators), and any conclusions about safety must be regarded as premature. The same concern applies to other colloids, with a 2012 systematic review making precisely this point about gelatins. ${ }^{[4]}$

Both sets of correspondents also argue that HES should still be available as there are no appropriate substitutes. HES trials have provided powerful signals of renal harm and possible mortality concerns in specific clinical contexts. To argue for their ongoing use in other contexts where safety information is deficient would only make sense if there were clear evidence of important clinical benefits. Most of the efficacy evidence put forward by the correspondents is for surrogate endpoints, is based on small trials, or involves trial designs highly susceptible to bias. The meta-analysis by Li et al. ${ }^{[5]}$ on the use of HES as preload in caesarean section patients is a case in point the primary endpoint of hypotension is not defined, in no included trial was there a clear description of allocation concealment, and the hypotension rate in the control groups varies from $24 \%$ to $90 \%$, raising concerns about heterogeneity despite the Q statistic. Also, in spite of the authors' contention, the funnel plot may well show evidence of publication bias.

In conclusion, we stand by our contention that South African clinicians should think carefully when prescribing HES, and we look forward to further regulatory guidance from both international bodies and the South African MCC on the ongoing use of these products.

\section{Andy Parrish}

Department of Internal Medicine, Frere and Cecilia Makiwane Hospitals, East London; Walter Sisulu University, Mthatha; Rhodes University, Grahamstown; and the University of Cape Town, South Africa

andygp@mweb.co.za

\section{Marc Blockman}

Department of Internal Medicine, Division of Clinical Pharmacology, Groote Schuur Hospital and University of Cape Town, South Africa

1. Position statement by the Faculty of Intensive Care Medicine, the Royal College of Anaesthetists, the Intensive Care Society and the College of Emergency Medicine. http://www.rcoa.ac.uk/news-and-bulletin/ rcoa-news-and-statements/risk-benefit-of-hes-solutions-questioned-ema (accessed 29 July 2013).

2. Intravenous fluid therapy in adults in hospital. Draft for consultation. 14 May 2013. National Institute 2. Intravenous fluid therapy in adults in hospital. Draft for consultation. 14 May 2013. National Institute
for Health and Care Excellence. http://www.nice.org.uk/nicemedia/live/13298/63879/63879.pdf (accessed 29 July 2013).

3. Protocols, physiology and trials of hydroyethylstarch. N Engl J Med 2012;367:1265-1267. [http:// 3. Protocols, physiology and trials of hydr
dx.doi.org/10.1056/NEJMc12099054]

4. Thomas-Rueddel DO, Vlasakov V, Reinhart K, et al. Safety of gelatin for volume resuscitation - a systematic review and meta-analysis. Intensive Care Medicine 2012;38:1134-1142. [http://dx.doi. org/10.1007/s00134-012-2560-X 5]

5. Li L, Zhang Y, Tan Y, Xu S. Colloid or crystalloid solution on maternal and neonatal hemodynamics for cesarean section: A meta-analysis of randomized controlled trials. J Obstet Gynaecol Res 2013;39(5):932-941. [http://dx.doi.org/10.1111/jog.12001]

S Afr Med J 2013;103(9):591-595. DOI:10.7196/SAMJ.7283 